\section{SUPPORTING YOUR NEEDS AS HEALTH PROFESSIONALS}

Johnson \& Johnson, the makers of LISTERINE, are delighted to bring you a new CPD programme, with the aim of supporting the ongoing needs of dental healthcare professionals in improving and maintaining oral health for their patients.

Through their programme, Johnson \& Johnson aim to deliver recent clinical findings, useful information for in-practice management of oral diseases, and patient communication strategies for improved preventive home care, as well as considering the effects of lifestyle and other external influences on your patients' oral health.

Visit www.listerineprofessional.co.uk/CPD-educational-programme to earn verifiable hours from Johnson and Johnson's launch pieces, including:

- Periodontal disease prevention: top tips

- A lecture with Professor Anthony Roberts: Understanding, identifying and managing the periodontal disease process

- Oral diseases: mouthwash use and the prevention of plaque, gingivitis and caries

- Overcoming dental anxiety with the power of language.

Upon completing each module successfully, Johnson \& Johnson will send you a CPD certificate by email within 14 days.

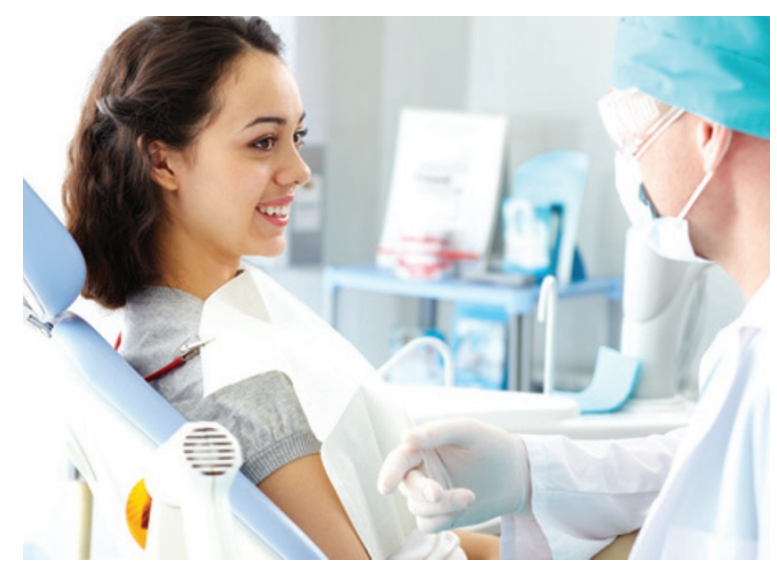

\title{
PERFECT TO REINFORCE ANY COMPOSITE RESTORATION
}

GC everX Posterior is a fibrereinforced composite designed to be used as dentine replacement, in conjunction with a conventional composite such as G-ænial Posterior used as enamel replacement. The short fibres of GC everX Posterior will make it a perfect sub-structure to reinforce any composite restoration in large size cavities. Fibres will also prevent and stop crack propagation through the filling, which is considered to be the main cause of composite failures. Thanks to its unique properties, GC everX Posterior opens new possibilities for restorations of extensive cavities at chairside and is the answer to the growing demand for an economic restorative alternative for big size cavities. Its properties include:

- Short fibres prevent and stop the progression of fracture in the restoration

- Fracture toughness equivalent to dentine and almost double to any composite will bring unsurpassed strength to the restoration

- 4 to $5 \mathrm{~mm}$ increments can be cured simultaneously

- Reliable bond to overlaying composite as well as to the tooth substance.

Indications: Reinforcing material for direct composite restorations, especially in large posterior cavities, including: cavities with three surfaces or more to be restored; cavities with missing cusps; deep cavities (including class I, II and endodontically treated teeth); cavities after amalgam replacement (especially since amalgam placement often lead to initiation of crack and cusp fracture); and cavities where onlays and inlays would also be indicated.

For further information contact GC UK on 01908218999.

\section{GIVE YOUR RESTORATIONS A LIFE-LIKE LUSTRE}

Trust the Sof-Lex spiral finishing and polishing wheels by $3 \mathrm{M} \mathrm{ESPE}$ to give your restorations a life-like lustre.

The two-step polishing system allows you to work quickly with the flexible spiral wheels - which are effective from any angle. The finishing wheel (beige) prepares the surface for polishing, while the superfine polishing wheel (white) creates a high-gloss finish.

Deliver the results your patients expect with the Sof-Lex spiral finishing and polishing wheels from 3M ESPE.

For more information, call 0845 6025094 or visit www.3Mespe. co.uk. 3M, ESPE and Sof-Lex are trademarks of the 3M Company.

\section{A COMPLETE SOLUTION FOR ALL AESTHETIC CERAMIC INDICATIONS}

The pre-shaded highly translucent Zirlux FC2 zirconium oxide system enables dental technicians to make aesthetic monolithic restorations efficiently and reliably for a wide range of indications, up to a 14-element bridge. This unique system comprises round blanks in five colours and ten shade pastes and six modifiers. With these pre- shaded round blanks and the paint-over characterisation and glazing kit, the dental technician can manufacture restorations in all 16 Vita shades (A-D) as well as bleach shades, without the need to dip them in coloured liquids, and therefore achieve an optimal aesthetic result.

Zirlux FC2 is available in the form of $98.5 \mathrm{~mm}$ and $100 \mathrm{~mm}$ round blanks for in-house fabrication. Furthermore, the Zirlux LC ceramic system and the Zirlux LC pressed ceramic are perfectly matched to the Zirlux FC2 round blanks.
With the Zirlux FC2 Henry Schein exclusively markets a zirconium oxide system for all aesthetic indications. Henry Schein offers selected laboratories the possibility to take part in the Zirlux partner programme. These partner laboratories support Henry Schein with the marketing of the Zirlux brand for dentists.

For further information, visit www.zirlux.co.uk. 